\title{
Erratum to: Predicting One and Three Month Postoperative Somatic Concerns, Psychological Distress, and Maladaptive Eating Behaviors in Bariatric Surgery Candidates with the Minnesota Multiphasic Personality Inventory-2 Restructured Form (MMPI-2-RF)
}

Ryan J. Marek • Yossef S. Ben-Porath • Julie Merrell •

Kathleen Ashton • Leslie J. Heinberg

Published online: 11 February 2014

(C) Springer Science+Business Media New York 2014

Erratum to: OBES SURG

DOI 10.1007/s11695-013-1149-y

A column in Table 3 of the published article had an incorrect column heading. The sixth column from the left (fifth from the right) should be labeled "Psychological distress month 3". Please see the corrected Table 3. 10.1007/s11695-013-1149-y.

R. J. Marek $(\bowtie) \cdot$ Y. S. Ben-Porath

Department of Psychology, Kent State University, Kent, OH 44242 ,

USA

e-mail: rmarek1@kent.edu

J. Merrell $\cdot$ K. Ashton $\cdot$ L. J. Heinberg

Cleveland Clinic Lerner College of Medicine, 9500 Euclid Avenue/

M61, Cleveland, OH 44195, USA 
Table 3 Correlations between the MMPI-2-RF Substantive Scales with the latent postoperative constructs

MMPI-2-RF Substantive Scales
Somatic Somatic $z$-test Psychological Psychological $z$-test concerns concerns month 1 month 3

distress distress

Maladaptive Maladaptive $z$-test eating eating behaviors behaviors month 1 month 3

\begin{tabular}{|c|c|c|c|c|c|c|c|c|c|}
\hline \multicolumn{10}{|l|}{ Higher-Order Scales } \\
\hline Emotional/Internalizing Dysfunction (EID) & 0.05 & 0.19 & $2.06^{*}$ & $0.31^{* *}$ & $0.42^{* *}$ & 1.84 & -0.06 & $0.27^{* *}$ & $4.88^{* *}$ \\
\hline Thought Dysfunction (THD) & 0.07 & 0.00 & 1.02 & 0.04 & $0.17^{*}$ & 1.91 & 0.08 & $0.30^{* * *}$ & $3.32^{* *}$ \\
\hline Behavioral/Externalizing Dysfunction (BXD) & -0.01 & 0.03 & 0.58 & 0.09 & 0.07 & 0.29 & $0.13^{*}$ & $0.36^{* *}$ & $3.56^{* *}$ \\
\hline \multicolumn{10}{|l|}{ Restructured Clinical (RC) Scales } \\
\hline Demoralization (RCd) & 0.07 & $0.21^{*}$ & $2.07^{*}$ & $0.34^{* *}$ & $0.43^{* *}$ & 1.53 & -0.06 & $0.25^{* *}$ & $4.57^{* * *}$ \\
\hline Somatic Complaints (RC1) & $0.27^{* *}$ & $0.30^{* * *}$ & 0.47 & 0.11 & $0.19^{* *}$ & 1.19 & -0.07 & $\overline{0.05}$ & 1.74 \\
\hline Low Positive Emotions (RC2) & 0.02 & 0.07 & 0.73 & $0.18^{* *}$ & $0.22^{* *}$ & 0.60 & -0.08 & $0.24^{* *}$ & $4.71^{* *}$ \\
\hline Cynicism (RC3) & 0.05 & 0.08 & 0.44 & 0.01 & 0.12 & 1.60 & 0.06 & 0.08 & 0.29 \\
\hline Antisocial Behaviors (RC4) & 0.06 & 0.10 & 0.58 & $0.19^{* *}$ & $0.17^{*}$ & 0.30 & 0.16 & $\underline{0.37^{* *}}$ & $3.29^{* * *}$ \\
\hline Ideas of Persecution (RC6) & 0.11 & 0.12 & 0.15 & 0.10 & $0.19^{*}$ & 1.33 & 0.04 & $0.41^{* *}$ & $5.73^{* * *}$ \\
\hline Dysfunctional Negative Emotions (RC7) & 0.07 & $0.19^{*}$ & $1.77^{*}$ & $0.29^{* *}$ & $0.39^{* *}$ & 1.64 & 0.05 & $0.30^{* *}$ & $3.76^{* *}$ \\
\hline Aberrant Experiences (RC8) & 0.09 & 0.04 & 0.73 & 0.08 & $0.19^{* *}$ & 1.62 & 0.05 & -0.01 & 0.87 \\
\hline Hypomanic Activation (RC9) & 0.03 & 0.05 & 0.29 & 0.07 & $0.16^{*}$ & 1.32 & $0.17^{*}$ & 0.13 & 0.59 \\
\hline \multicolumn{10}{|l|}{ Somatic Complaints Specific Problems Scales } \\
\hline Malaise (MLS) & $0.15^{* *}$ & 0.10 & 0.74 & $0.25^{* *}$ & $0.33^{* *}$ & 1.27 & -0.07 & $0.31^{* *}$ & $5.66^{* *}$ \\
\hline Gastrointestinal Complaints (GIC) & $0.13^{*}$ & 0.12 & 0.15 & 0.03 & $0.34^{* *}$ & $4.69^{* * *}$ & -0.03 & $0.37^{* *}$ & $6.06^{* * *}$ \\
\hline Head Pain Complaints (HPC) & $0.23^{* *}$ & $0.27^{* *}$ & 0.62 & $0.15^{*}$ & $0.25^{* *}$ & 1.51 & -0.09 & 0.10 & $2.76^{* *}$ \\
\hline Neurological Complaints (NUC) & $0.22^{* * *}$ & $0.20^{*}$ & 0.30 & 0.07 & 0.10 & 0.44 & 0.05 & 0.07 & 0.29 \\
\hline Cognitive Complaints (COG) & $00.13^{*}$ & $\overline{0.10}$ & 0.44 & $0.19^{* *}$ & $0.35^{* *}$ & $2.51^{*}$ & -0.02 & $0.33^{* *}$ & $5.25^{* *}$ \\
\hline \multicolumn{10}{|l|}{ Internalizing Specific Problems Scales } \\
\hline Suicidal/Death Ideation (SUI) & -0.02 & $-0.35^{* *}$ & $5.00^{* * *}$ & $0.17^{* *}$ & 0.00 & $2.49^{*}$ & 0.07 & 0.16 & 1.32 \\
\hline Helplessness/Hopelessness (HLP) & 0.04 & -0.03 & 1.01 & $15^{* *}$ & 0.13 & 0.30 & -0.06 & 0.14 & $2.91^{* *}$ \\
\hline Self-Doubt (SFD) & 0.00 & 0.10 & 1.45 & $\underline{0.31^{* *}}$ & $0.34^{* *}$ & 0.49 & -0.05 & $0.22^{*}$ & $3.96^{* * *}$ \\
\hline Inefficacy (NFC) & 0.10 & 0.10 & 0.00 & $0.26^{* *}$ & $0.30^{* *}$ & 0.63 & 0.00 & 0.14 & $2.04^{*}$ \\
\hline Stress/Worry (STW) & 0.09 & $0.28^{*}$ & $2.86^{* *}$ & $0.32^{* *}$ & $0.34^{* *}$ & 0.33 & -0.05 & $0.27^{*}$ & $4.73^{* *}$ \\
\hline Anxiety (AXY) & $0.19^{*}$ & $0.27^{* *}$ & 1.22 & $0.30^{* * *}$ & $0.39^{* *}$ & 1.48 & 0.05 & $0.48^{* *}$ & $6.85^{* *}$ \\
\hline Anger Proneness (ANP) & 0.05 & 0.17 & 1.76 & $0.16^{* *}$ & $0.25^{* *}$ & 1.36 & -0.00 & 0.18 & $2.64^{* *}$ \\
\hline Behavior-Restricting Fears (BRF) & $0.14^{* *}$ & $0.18^{*}$ & 0.59 & $0.14^{*}$ & $\overline{0.16^{*}}$ & 0.30 & -0.02 & 0.14 & $2.33^{*}$ \\
\hline Multiple Specific fFears (MSF) & $0.18^{* *}$ & 0.04 & $2.06 * *$ & 0.03 & 0.04 & 0.14 & -0.02 & 0.07 & 1.31 \\
\hline \multicolumn{10}{|l|}{ Externalizing Specific Problems Scales } \\
\hline Juvenile Conduct Problems (JCP) & 0.01 & 0.08 & 10.02 & 0.10 & 0.05 & 0.73 & 0.07 & $\underline{0.32^{*}}$ & $3.79^{* *}$ \\
\hline Substance Abuse (SUB) & -0.07 & 0.11 & 20.61 & 0.10 & -0.02 & 1.74 & 0.13 & $\underline{0.23}$ & 1.50 \\
\hline Aggression (AGG) & -0.01 & 0.03 & 0.58 & $0.13^{*}$ & $0.17^{*}$ & 0.59 & $0.13^{*}$ & $\overline{0.13}$ & 0.00 \\
\hline Activation (ACT) & 0.05 & 0.01 & 0.58 & $0.16^{* *}$ & $0.25^{* *}$ & 1.36 & 0.12 & 0.02 & 1.46 \\
\hline \multicolumn{10}{|l|}{ Interpersonal Specific Problems Scales } \\
\hline Family Problems (FML) & 0.06 & 0.17 & 1.62 & $0.20^{* * *}$ & $0.32^{* *}$ & 1.87 & 0.03 & 0.16 & 1.90 \\
\hline Interpersonal Passivity (IPP) & 0.04 & 0.00 & 0.58 & 0.11 & $\overline{-0.05}$ & $2.32^{*}$ & -0.06 & 0.06 & 1.74 \\
\hline Social Avoidance (SAV) & 0.01 & 0.02 & 0.14 & $0.18^{* *}$ & $0.26^{* *}$ & 1.22 & -0.10 & 0.12 & $3.20^{* * *}$ \\
\hline Shyness (SHY) & -0.08 & 0.12 & $2.91^{* * *}$ & 0.12 & $0.24^{* *}$ & 1.80 & -0.05 & 0.03 & 1.16 \\
\hline Disaffiliativeness (DSF) & -0.00 & -0.02 & 0.29 & 0.08 & 0.06 & 0.29 & -0.02 & 0.03 & 0.72 \\
\hline \multicolumn{10}{|l|}{ Interest Scales } \\
\hline Aesthetic-Literary Interests (AES) & 0.07 & 0.07 & 0.00 & 0.04 & 0.14 & 1.46 & $0.21^{* *}$ & 0.05 & $2.36^{*}$ \\
\hline Mechanical-Physical Interests (MEC) & -0.09 & -0.12 & 0.44 & 0.11 & $-0.17^{*}$ & $4.09^{* * *}$ & $0.12^{*}$ & -0.03 & $2.18^{*}$ \\
\hline \multicolumn{10}{|l|}{ Personality Psychopathology Five (PSY-5) Scales } \\
\hline Aggressiveness-revised (AGGR-r) & -0.07 & 0.03 & 1.45 & $-0.14^{*}$ & -0.02 & 1.75 & 0.13 & -0.01 & $2.04^{*}$ \\
\hline Psychoticism-revised (PSYC-r) & 0.06 & -0.02 & 1.16 & 0.09 & $0.19^{* *}$ & 1.48 & 0.06 & $0.28^{* *}$ & $3.30^{* * *}$ \\
\hline
\end{tabular}


Table 3 (continued)

MMPI-2-RF Substantive Scales
Somatic Somatic $z$-test concerns concerns

month 1 month 3
Psychological Psychological z-test distress month 1
Maladaptive Maladaptive $z$-test eating behavior month 1 behaviors month 3

\begin{tabular}{|c|c|c|c|c|c|c|c|c|c|}
\hline Disconstraint-revised (DISC-r) & -0.05 & -0.08 & 0.44 & 0.07 & -0.03 & 1.45 & $0.15^{* *}$ & 0.27 & 1.82 \\
\hline $\begin{array}{l}\text { Negative Emotionality/Neuroticism-revised } \\
\text { (NEGE-r) }\end{array}$ & 0.09 & $0.22^{*}$ & 1.93 & $0.32^{* *}$ & $0.40^{* *}$ & 1.33 & -0.04 & $\underline{0.31^{* *}}$ & $5.22^{*}$ \\
\hline $\begin{array}{l}\text { Introversion/low Positive Emotionality- } \\
\text { revised (INTR-r) }\end{array}$ & 0.00 & 0.02 & 0.29 & 0.08 & $0.16^{*}$ & 1.18 & -0.12 & 0.16 & $4.08^{*}$ \\
\hline
\end{tabular}

$M M P I-2-R F$ Minnesota Multiphasic Personality Inventory-2 Restructured Form

${ }^{*} p<0.05,{ }^{* *} p<0.01 ; r \geq 0.20$ (underlined); significant $z$ test (bold) 